\title{
Magnetic increases with central current sheets: observations with Parker Solar Probe
}

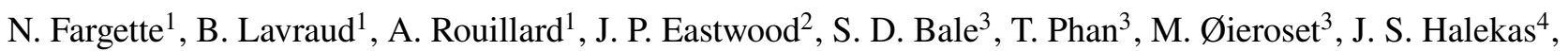 \\ J. Kasper ${ }^{5}$, M. Berthomier ${ }^{6}$, A. W. Case ${ }^{7}$, K. E. Korreck ${ }^{7}$, D. E. Larson ${ }^{3}$, P. Louarn ${ }^{1}$, D. Malaspina ${ }^{8,9}$, M. Pulupa ${ }^{3}$,
} M. L. Stevens ${ }^{7}$, P. L. Whittlesey ${ }^{3}$, R. J. MacDowall ${ }^{10}$, K. Goetz ${ }^{11}$, P. R. Harvey ${ }^{3}$, T. Dudok de Wit ${ }^{12}$, and J. W. Bonnell ${ }^{3}$

${ }^{1}$ Institut de Recherche en Astrophysique et Planétologie, CNRS, UPS, CNES, Toulouse, France e-mail: nfargette@irap.omp.eu

2 The Blackett Laboratory, Department of Physics, Imperial College London, London, UK

${ }^{3}$ Space Sciences Laboratory, University of California, Berkeley, Berkeley, CA, USA

${ }^{4}$ Department of Physics and Astronomy, University of Iowa, Iowa City, Iowa, USA

${ }^{5}$ Climate and Space Sciences and Engineering, University of Michigan, Ann Arbor, MI, USA

${ }^{6}$ Laboratoire de Physique des Plasmas, CNRS, Sorbonne Universite, Ecole Polytechnique, Observatoire de Paris, Universite Paris-Saclay, Paris, France

${ }^{7}$ Smithsonian Astrophysical Observatory, Cambridge, Massachusetts, USA

8 Department of Astrophysical and Planetary Sciences, University of Colorado, Boulder, CO, USA

${ }_{9}^{9}$ Laboratory for Atmospheric and Space Physics, University of Colorado, Boulder, CO, USA

10 Solar System Exploration Division, NASA/Goddard Space Flight Center, Greenbelt, Maryland 20771, USA

${ }^{11}$ School of Physics and Astronomy, University of Minnesota, Minneapolis, Minnesota 55455, USA

12 LPC2E, CNRS and University of Orléans, 3 Avenue de la Recherche Scientifique, 45071 Orléans, France

Received 15 August 2020 / Accepted 23 October 2020

\begin{abstract}
Aims. We report the observation by Parker Solar Probe (PSP) of magnetic structures in the solar wind that present a strong peak in their magnetic field magnitude with an embedded central current sheet. Similar structures have been observed, either at the Earth's magnetopause and called interlinked flux tubes, or in the solar wind and called interplanetary field enhancements.

Methods. In this work, we first investigate two striking events in detail; one occurred in the regular slow solar wind on November 2 , 2018 and the other was observed during a heliospheric current sheet crossing on November 13, 2018. They both show the presence of a central current sheet with a visible ion jet and general characteristics consistent with the occurrence of magnetic reconnection. We then performed a survey of PSP data from encounters 1 to 4 and find 18 additional events presenting an increase in the magnetic field magnitude of over $30 \%$ and a central current sheet. We performed a statistical study on the 20 "magnetic increases with central current sheet" (MICCS), with 13 observed in the regular slow solar wind with a constant polarity (i.e., identical strahl direction), and 7 which were specifically observed near a heliospheric current sheet crossing.

Results. We analyze and discuss the general properties of the structures, including the duration, location, amplitude, and magnetic topology, as well as the characteristics of their central current sheet. We find that the latter has a preferential orientation in the TN plane of the RTN frame. We also find no significant change in the dust impact rate in the vicinity of the MICCS under study, leading us to conclude that dust probably plays no role in the MICCS formation and evolution. Our findings are overall consistent with a double flux tube-configuration that would result from initially distinct flux tubes which interact during solar wind propagation.
\end{abstract}

Key words. solar wind - magnetic reconnection - magnetic fields

\section{Introduction}

Significant fluctuations in the amplitude $\|\boldsymbol{B}\|$ of the interplanetary magnetic field (IMF) are typically due to specific magnetic structures associated with both small- and large-scale disturbances of the ambient solar wind plasma. At large scales, typical perturbations are associated with coronal mass ejections (CMEs) or compression regions, such as corotating interaction regions (CIRs), typically as the result of stream interactions, the creation of pressure waves and shocks, etc. At smaller scales, disturbances in the $\boldsymbol{B}$ field amplitude can stem from either velocity shears and turbulence in general (e.g., Bruno \& Carbone 2013), or from small scale magnetic structures. The latter typically consists of the following : flux tubes, as in the view of Borovsky
(2008), for example, where they constitute the very texture of the solar wind; flux ropes often observed close to the heliospheric current sheet (HCS); and interplanetary field enhancements.

The specific kind of disturbance in the solar wind magnetic field called interplanetary field enhancements (IFEs) were first reported by Russell et al. (1984) from observations with the Pioneer Venus spacecraft. The main signature of these structures is a strong peak in the amplitude of the magnetic field, lasting from minutes to several hours, that is very distinguishable from the background field. The second main feature that they present is a thin current sheet in their core. They propagate at the solar wind speed (Russell et al. 2010a) and were observed from 0.3 to 1 AU with several missions, such as Helios, STEREO, ACE, or Wind (Russell et al. 2010b, Lai et al. 2015). These structures were 
proposed to have been formed through an interaction between the interplanetary magnetic field and dust trails of asteroids in the solar wind (Russell 1990, Jones et al. 2003a,b). This explanation is still debated within the scientific community, as the physical process to transform small dust particles to a strong $\boldsymbol{B}$ disturbance remains unclear. Mass loading of the solar wind by cometary dust trails was put forward as a possible explanation, but it was found to be insufficient (Mann et al. 2010).

Interestingly, structures that are somewhat similar to IFEs are observed at the Earth's magnetopause and were, until recently, interpreted as flux transfer events (FTEs). FTEs are transient phenomena that frequently occur at the Earth's dayside magnetopause, resulting from a reconnection at the subsolar point between the solar wind and the magnetosphere (e.g., Russell \& Elphic 1979, Southwood et al. 1988, Lee \& Fu 1985). They are usually thought of as helical structures (flux ropes) and hence their classical in situ signatures are an enhancement in their core magnetic field strength and a bipolar signature in the magnetic field component, which is normal for the magnetopause surface. However, recent studies found that certain FTEs present a thin current sheet in their center, concluding that they did not fit a flux rope configuration (Øieroset et al. 2016, 2019, Kacem et al. 2018, Fargette et al. 2020, Russell \& Qi 2020). In these events that strongly resemble the solar wind IFEs, the very high time resolution of Magnetospheric Multiscale (MMS) mission data allowed for magnetic reconnection to be resolved at the current sheets, including the clear identification of ion jets (Øieroset et al. 2016, 2019, Kacem et al. 2018, Fargette et al. 2020). These structures were interpreted as interlaced flux tubes - as originally proposed by Nishida (1989) and Hesse et al. (1990), and first reported in the Cluster mission's observations by Louarn et al. (2004) - with the reconnecting current sheet separating two magnetically disconnected regions. This interpretation was further backed in a statistical study (Fargette et al. 2020), supporting that these FTEs with a central current sheet (1) were usually constituted of two regions with a distinct magnetic connectivity, and (2) formed under IMF conditions with a strong transverse $B_{Y}$ component (in addition to being southward). Indeed, a strong IMF $B_{Y}$ may lead to a complex configuration of the reconnection sites at the dayside magnetopause, and this multiplication of possible reconnection locations can lead to the creation of interlaced flux tubes. This also agrees with numerical results: When imposing a strong $B_{Y}$ in the IMF, interlaced flux tubes are observed (Cardoso et al. 2013 and Farinas Perez et al. 2018), while Otto (1995) showed that magnetic reconnection is favored at the interface of such interlaced flux tubes.

In this paper we focus on structures observed by NASA's Parker Solar Probe (PSP) mission in solar wind. Traditionally, solar wind can be separated into two types of winds, fast and slow, which obviously differ in speed, but also in density, composition, and source regions at the Sun. The fast solar wind is a tenuous plasma that propagates at around $600 \mathrm{~km} \mathrm{~s}^{-1}$ from the Sun and can be traced back to coronal holes with open magnetic field lines in the low corona (e.g., Cranmer 2009). By contrast, the slow solar wind is denser and propagates at around $300 \mathrm{~km} \mathrm{~s}^{-1}$. Its origin is less clear and different source regions are identified and advocated for in the literature. They include (1) the boundary of coronal holes as well as low latitude coronal holes where flux tube expansion may accelerate the plasma (e.g., Wang 1994, Bale et al. 2019), and (2) the release of transients through magnetic reconnection at the tip of the coronal streamer (e.g., Lapenta \& Knoll 2005, Antiochos et al. 2011, Sanchez-Diaz et al. 2017).
In the heliosphere, the heliospheric current sheet (HCS) also plays an important role in structuring the solar wind. The HCS is a current sheet that originates at the tip of the helmet streamer (closed field lines of the Sun's magnetic field) and expands into the interplanetary medium. It separates zones of an opposite magnetic polarity connecting to each hemisphere of the Sun (e.g., Gosling et al. 1981). It is well identified in in situ data, by a reversal of the radial component of the magnetic field, and the change in the relative propagation direction of the strahl, a high energy electron population traveling outward from the Sun. Near the HCS, we find a region of the heliosphere that is characterized by a low beta plasma with strahl drop out and complex magnetic configurations. It is a favored place for the process of magnetic reconnection and the formation of potential flux ropes (Lavraud et al. 2020). In this paper, we refer to regular slow and fast solar wind for plasma exhibiting no complex magnetic configuration with a uniform strahl propagating outward from the Sun. By contrast, we refer to nearby HCS for events that are observed in the direct vicinity or inside the HCS, as identified on the basis of plasma, the magnetic field, and strahl properties, as explained above.

The primary goal of this paper is to report the observation of structures that show significant magnetic field increases by the PSP mission in the solar wind, between 0.1 and $0.6 \mathrm{AU}$ (20-120 Rs). In this study we choose to use a new descriptive term for the observed events, and from now on refer to them as magnetic increases with central current sheet (MICCS). This choice was made in order to avoid confusion with other phenomena that lead to interplanetary field enhancement - such as ICMEs and other small-scale flux ropes or compression regions - and so as to avoid any bias regarding their still debated origin. This paper is organized as follows. In Sect. 2 we detail the mission and instrumentation used. Sections 3 and 4 present two events that occur during the first orbit of PSP in different contexts, the first in regular slow solar wind (Eq. (3)) and the other near the HCS (Eq. (4)). Both display clear reconnection signatures in their central current sheet. In Sect. 5 we preform an analysis of 20 structures found through encounters 1 to 4 . We detail the selection process (Sect. 5.1), their general properties (Sect. 5.2), discuss the features of their central current sheet (Sects 5.3, 5.4), the evolution of the pitch angle distributions (Sect. 5.5), their possible relation with the radial inversions of the magnetic field commonly known as switchbacks (e.g., Kasper et al. 2019, Bale et al. 2019, Dudok de Wit et al. 2020, Horbury et al. 2020) (Sect. 5.6), and analyze the dust impact rate (Sect. 5.7). Finally we discuss the possible formation model for these events in Sect. 6 and summarize our results in Sect. 7.

\section{Mission and instrumentation}

The Parker Solar Probe mission was launched by NASA in August 2018 and has been transmitting unprecedented data since its first perihelion in November 2018. The first three orbits brought the spacecraft down to a distance of $35.6 R_{\mathrm{S}}$ from the Sun (0.166 AU); and the fourth one reached 27.8 $R_{\mathrm{s}}(0.130 \mathrm{AU})$. In this study we analyze the magnetic field, electric field, and particle data taken by the different in situ instruments during these first four encounters. Magnetic and electric field data are provided by the FIELDS instrument suite (Bale et al. 2016) and the particle data by the Solar Wind Electrons Alphas and Protons (SWEAP) instrument suite (Kasper et al. 2016). The latter includes plasma moments from the Solar Probe Cup (SPC; Case et al. 2020) and electron pitch angle distributions from the 


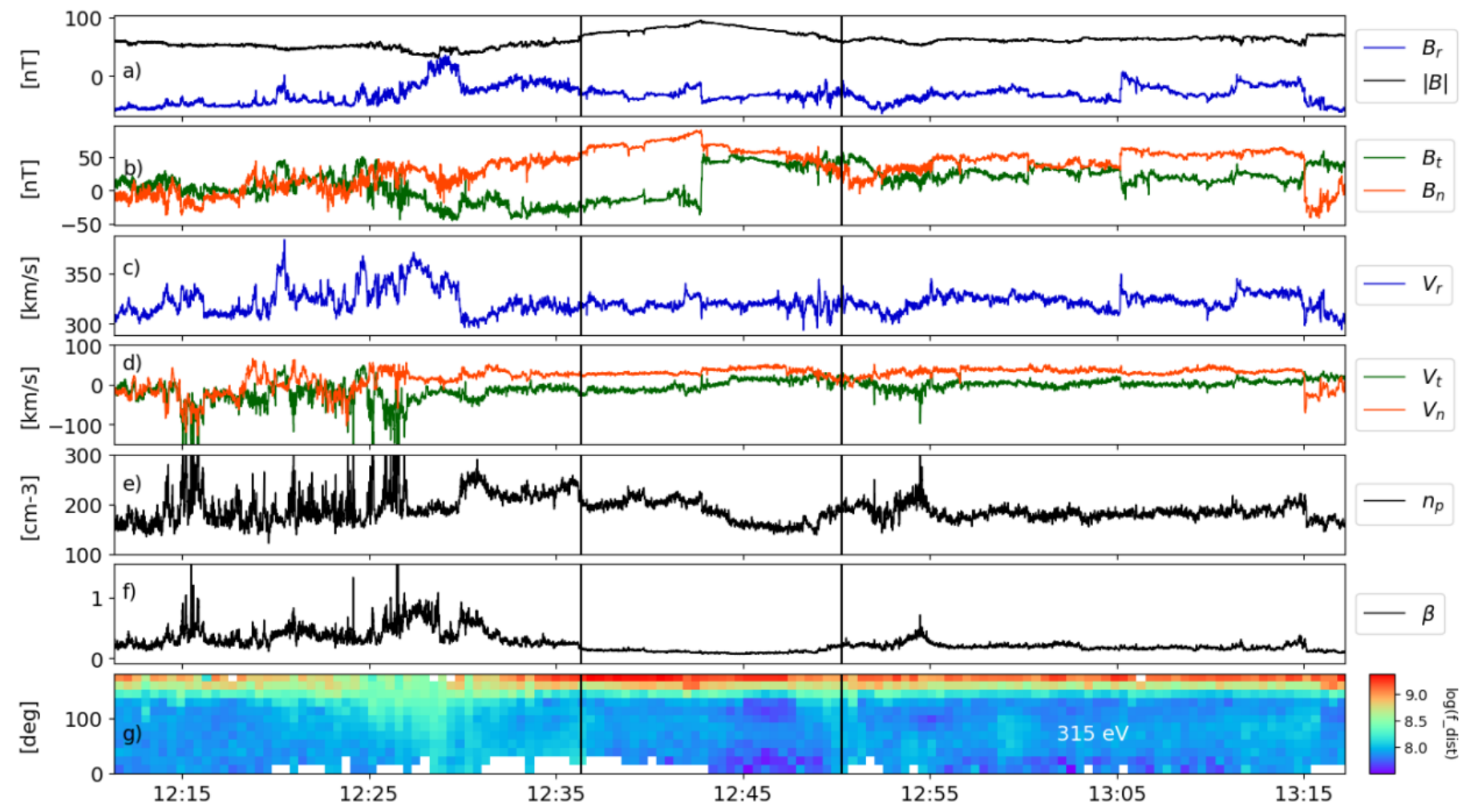

Fig. 1. Event from November 2, 2018 (12:36 to 12:50, boundaries in black vertical lines). From top to bottom: (a) magnetic field's amplitude $|B|$ and its radial component $B_{\mathrm{r}} ;(b)$ tangential and normal magnetic field components $B_{\mathrm{t}}$ and $B_{\mathrm{n}} ;(c)$ radial ion velocity $V_{\mathrm{r}} ;(d)$ tangential and normal ion velocity $V_{\mathrm{t}}$ and $V_{\mathrm{n}} ;(e)$ proton density $n_{\mathrm{p}} ;(f)$ plasma $\beta$; and $(g)$ pitch angle distribution of electrons at $315 \mathrm{eV}$, with $f_{\text {dist }}$ being the phase space density in $\mathrm{s}^{3} \mathrm{~km}^{6}$.

Solar Probe ANalyzers (SPANs; Whittlesey et al. 2020). Data are shown in the RTN frame of reference, with $\boldsymbol{R}$ (radial) being the Sun to spacecraft unit vector, $\boldsymbol{T}$ (tangential) being the cross product between the Sun's spin axis and $\boldsymbol{R}$, and $\boldsymbol{N}$ (normal) completes the direct orthogonal frame.

\section{Event of November 2, 2018}

\subsection{Context}

During its first encounter with the Sun, PSP observed a striking event, such as those described in the Introduction, with a clear and smooth magnetic strength increase and an embedded reconnecting current sheet. The structure was observed at $44 R_{\mathrm{s}}$ (0.2 AU) from the Sun on November 2, 2018, when PSP was probably magnetically connected to a small equatorial coronal hole (Bale et al. 2019, Badman et al. 2020, Réville et al. 2020).

In Fig. 1 we display the magnitude and components of the magnetic field $(1 \mathrm{a}, 1 \mathrm{~b})$, the ion velocity $(1 \mathrm{c}, 1 \mathrm{~d})$, the ion density (1e), the plasma $\beta$ (ratio between ion thermal plasma pressure and magnetic pressure, 1f), and the pitch angle distribution (PAD) for electrons at $315 \mathrm{eV}(1 \mathrm{~g})$. Vertical black lines frame the event of interest. To reduce instrumental artifacts and improve data visualization, we applied a median filter of $1.5 \mathrm{~s}$ on the SPC moments; nevertheless, some instrumental variations remain. The event lasted 14 min from 12:36 to $12: 50$, and moved at $321 \mathrm{~km} \mathrm{~s}^{-1}$ in the radial direction on average. The maximum magnetic field was measured at $94 \mathrm{nT}$ at 12:42:50 while the background field was around $65 \mathrm{nT}$ (45\% increase). The proton density fluctuates around the central current sheet with a mean value of $213 \pm 9 \mathrm{~cm}^{-3}$ before it and $172 \pm 15 \mathrm{~cm}^{-3}$ afterwards. The plasma $\beta$ parameter remains constant around 0.1 during the event. The supra-thermal electrons show a strahl mainly antiparallel to the magnetic field with a peak at a $180^{\circ}$ pitch angle $(1 \mathrm{~g})$, even though pitch angle scattering seems to occur right before the event between 12:20 and 12:30. The occasional lack of data (white pixels) near the $0^{\circ}$ pitch angle corresponds to directions that are affected by spacecraft effects and were thus removed.

\subsection{Central reconnection jet}

The central current sheet of the event is coincidental with the magnetic peak and was reported to reconnect by Phan et al. (2020), with a low shear of $55^{\circ}$, a guide field of 1.9 nT, and a distance to the X-line of $0.012 \mathrm{Rs}$. The ion jet is visible in all velocity components (1c, 1d). Figure 2 displays the magnetic field in the $l m n$ frame associated with the current sheet (2a) and the $l$ component of the ion velocity (2b). The $\operatorname{lmn}$ frame is obtained through a hybrid minimum-variance method (Gosling \& Phan 2013), which often works best for low-magnetic-shear current sheets. The current sheet normal is $\boldsymbol{n}=\left(\boldsymbol{B}_{1} \wedge \boldsymbol{B}_{2}\right) /\left\|\boldsymbol{B}_{1} \wedge \boldsymbol{B}_{2}\right\|$ where $\boldsymbol{B}_{1}$ and $\boldsymbol{B}_{2}$ are the boundary magnetic fields on both sides of the current sheet; $\boldsymbol{m}=\boldsymbol{l}^{\prime} \wedge \boldsymbol{n}$ where $\boldsymbol{l}^{\prime}$ is the direction of maximum variance of the magnetic field (Sonnerup \& Cahill 1967); and $\boldsymbol{l}=\boldsymbol{m} \wedge \boldsymbol{n}$ completes the orthogonal frame. When determining the direction of maximum variation $\boldsymbol{l}^{\prime}$, we checked that the ratio of the maximum to intermediate eigenvalue obtained through regular variance analysis was high enough. In this case, 

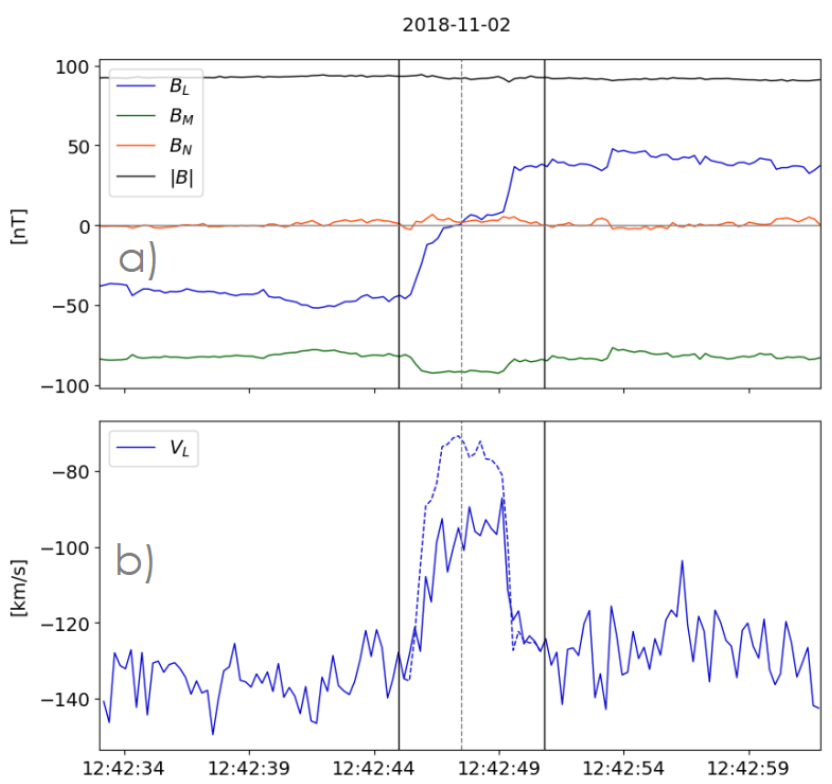

Fig. 2. Reconnection jet for the November 2, 2018 MICCS, framed by two vertical black lines (which are used as a reference for the Walén test). Top panel: magnetic field in the current sheets' Imn frame (see text for more details); bottom panel: $\boldsymbol{l}$ component of the ion velocity, as well as the $\boldsymbol{l}$ component predicted from the Walén test and plotted as a dashed blue line.

Table 1. Current sheet Imn orientation in the RTN frame for the November 2, 2018 reconnection jet event.

\begin{tabular}{cccc}
\hline \hline & $\mathrm{R}$ & $\mathrm{T}$ & $\mathrm{N}$ \\
\hline $\mathrm{l}$ & -0.35 & 0.90 & -0.27 \\
$\mathrm{~m}$ & 0.37 & -0.14 & -0.92 \\
$\mathrm{n}$ & -0.86 & -0.42 & -0.28 \\
\hline
\end{tabular}

the ratio was 39 , which largely ensures that $\boldsymbol{l}^{\prime}$ was accurately determined. The transformation matrix is displayed in Table 1 and is consistent with the discontinuity being mainly in the $\mathrm{T}$ direction.

In this frame, the variation of velocity observed in the $\boldsymbol{l}$ direction is $40 \mathrm{~km} \mathrm{~s}^{-1}$. In comparison, the dashed blue line in Fig. 2 is the predicted jet velocity, derived from the Walén relation for a rotational discontinuity: $\Delta \boldsymbol{v}_{\text {theory }}= \pm \Delta \boldsymbol{v}_{\mathrm{A}}$ where $\boldsymbol{v}_{\mathrm{A}}$ is the Alfvén velocity (e.g., Hudson 1970, Sonnerup et al. 1981). From this equation, we derived the theoretical velocity, starting from two states of reference on each side of the jet (vertical lines at 12h42:45.0 and 12h42:50.8 in Fig. 2) and going inward toward its center (dotted line at 12h42:47.5) with a positive correlation on the left side and a negative correlation on the right side. The theoretical velocity variation is found to be around $60 \mathrm{~km} \mathrm{~s}^{-1}$. This overestimation of the jet speed by the Walén relation is usual in observations, though not totally understood. It may be explained by the proximity to the X-line because in the ion diffusion region next to the reconnection site, the ion jet may not be fully developed. Phan et al. (2020) discuss several other possible explanations, such as non-ideal rotational discontinuities at the boundaries of the jet (e.g., Liu et al. 2011) or ion temperature anisotropy in the exhaust (Haggerty et al. 2018).

We note that this MICCS event occurs in the vicinity of some radial magnetic inversions commonly known as switchbacks (e.g., Kasper et al. 2019, Bale et al. 2019, Dudok de Wit
Table 2. Current sheet Imn orientation in the RTN frame for the November 13, 2018 reconnection jet event.

\begin{tabular}{cccc}
\hline \hline & $\mathrm{R}$ & $\mathrm{T}$ & $\mathrm{N}$ \\
\hline $\mathrm{l}$ & 0.59 & -0.48 & 0.65 \\
$\mathrm{~m}$ & 0.01 & -0.8 & -0.6 \\
$\mathrm{n}$ & 0.8 & 0.36 & -0.47 \\
\hline
\end{tabular}

et al. 2020, Horbury et al. 2020). From 12:18 to 13:18, the magnetic field deviates from its ground state, corresponding to a Parker spiral of $166^{\circ}$ (based on the average solar wind speed), and the radial magnetic field becomes positive once before the event (12:27) and once after (13:07) in what resemble two successive switchbacks. We discuss a possible link between MICCS and switchbacks in Sects. 5.6 and 6

\section{Event of November 13, 2018}

Another impressive event was observed on November 13, 2018 and is particularly interesting as PSP was crossing the HCS at this time (Szabo et al. 2020, Lavraud et al. 2020). It is displayed in Fig. 3 in the same format as in Fig. 1. The structure was observed at $62 R_{s}(0.3 \mathrm{AU})$ from the Sun and lasted $105 \mathrm{~min}$ from $09: 27$ to $11: 12$. The maximum magnetic field was measured at $41 \mathrm{nT}$ at 10:04, and its average value at the boundaries is around $24 \mathrm{nT}$ yielding a $71 \%$ increase. The wind speed was $350 \mathrm{~km} \mathrm{~s}^{-1}$ throughout the event; density was measured at $222 \mathrm{~cm}^{-3}$, and the mean $\beta$ was 0.28 . The main differences with the event described in Sect. 3 lie in both the location of the event (i.e., nearby or at the HCS in the sense described in the Introduction) and the PAD variations $(3 \mathrm{~g})$. We first observe a strahl drop out (8:45-9:15) consistent with PSP beginning to cross the HCS (Lavraud et al. 2020). Then within the MICCS, the electrons are first isotropic, until bidirectional electrons are observed on four samples during the first part of the event that precedes the central current sheet. The PAD appears to have larger bidirectional fluxes for the sample closest to the current sheet, but this may be the result of time aliasing; it cannot be confirmed without higher time resolution. The second part of the event is dominated by a unidirectional parallel strahl population, before the PAD becomes isotropic again after the trailing boundary of the event at 11:12.

As reported by Phan et al. (2020), the central current sheet of the MICCS also reconnects, with signatures such as the ion jet visible in the $\mathrm{R}$ and $\mathrm{N}$ velocity components (3c, 3d), an indent in the magnetic field's amplitude (3a), and a flux enhancement in the PAD of the supra-thermal electrons $(3 g)$; however, as previously mentioned, the apparent bidirectional PAD might be due to time aliasing. The $\operatorname{lmn}$ frame associated with the current sheet was obtained through a hybrid minimum variance analysis as before and is given in Table 2, with a ratio of maximum to intermediate eigen value related to $\boldsymbol{l}^{\prime}$ of 25 . We note that this time the $\boldsymbol{l}$ vector is evenly distributed on the three RTN directions.

\section{Statistical analysis}

\subsection{Selection process}

To obtain a more complete view of the MICCS events measured by Parker Solar Probe in the inner heliosphere, we performed a survey covering the first four encounters. Our selection criteria were based on a magnetic field increase over $30 \%$ and a 

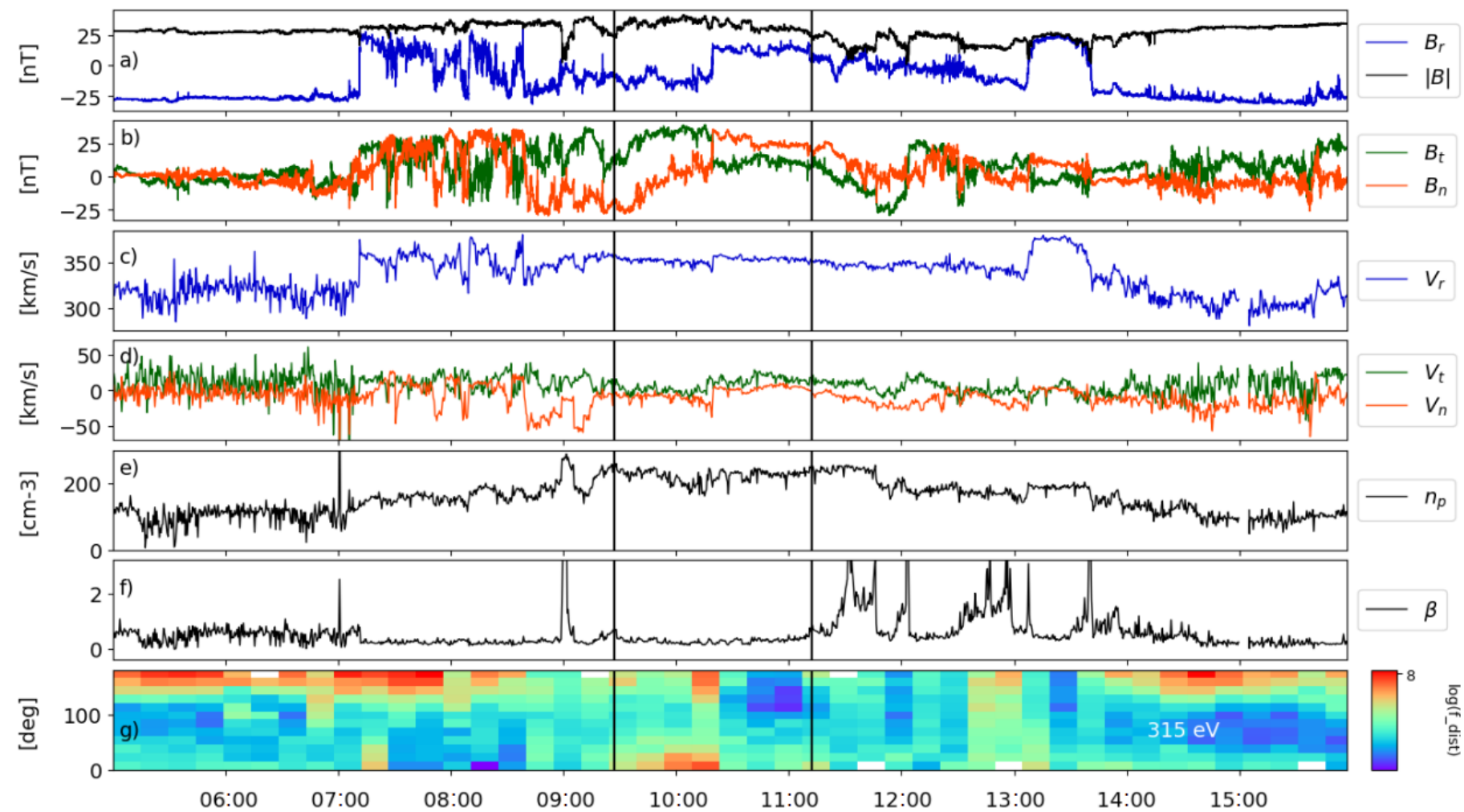

Fig. 3. Event from November 13, 2018 (09:27 to 11:21, boundaries in black vertical lines). The data are presented in the same manner as in Fig. 1.
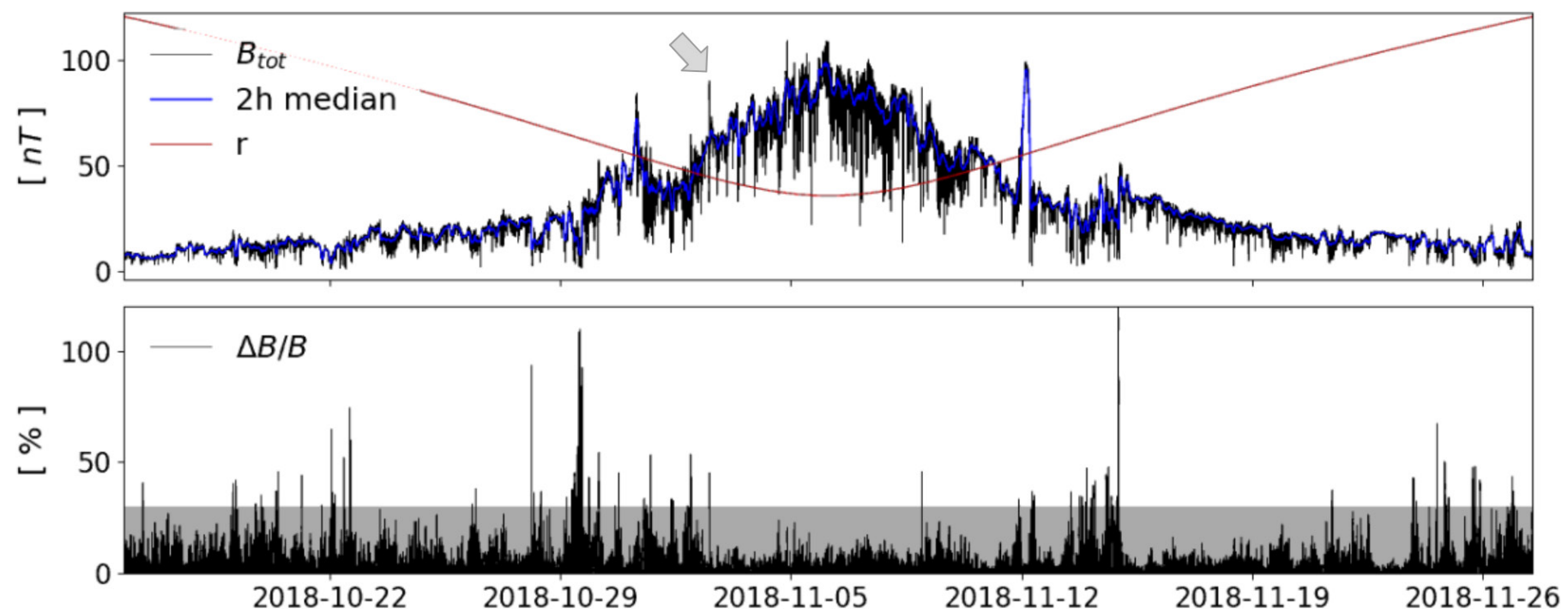

Fig. 4. Search process illustration. Top panel: magnetic field's amplitude for the first encounter $\left(B_{\text {tot }}\right)$, as well as a 2-h running median (in blue) and $r$ the radial distance to the Sun. Bottom panel: relative variation of $B_{\mathrm{tot}}$ regarding its $2 \mathrm{~h}$ running median, with emphasis on over $+30 \%$ relative variation. The gray arrow in the top panel indicates the event occurring on November 2, 2018, which is detailed in Sect. 3.

current sheet embedded in the structure. To automate the detection process, we searched for increases in the magnitude of the magnetic field relative to a background determined by computing a running median. This was repeated over several running median time windows of $0.5,2,4$, and $6 \mathrm{~h}$. Figure 4 illustrates this method for the 2 -h running median during encounter 1 . The top panel displays the magnetic field as well as the distance to the Sun $r$ (in $R_{s}$ ). The gray arrow indicates the event occurring on November 2, 2018 detailed in Sect. 3. The bottom panel highlights all the time intervals that we investigated in detail, as well as a gray shading to highlight events above $30 \%$ of the background. Upon visual inspection of the data, we determined if the event corresponds to a magnetic structure with an embedded current sheet. However, we noted that the running 

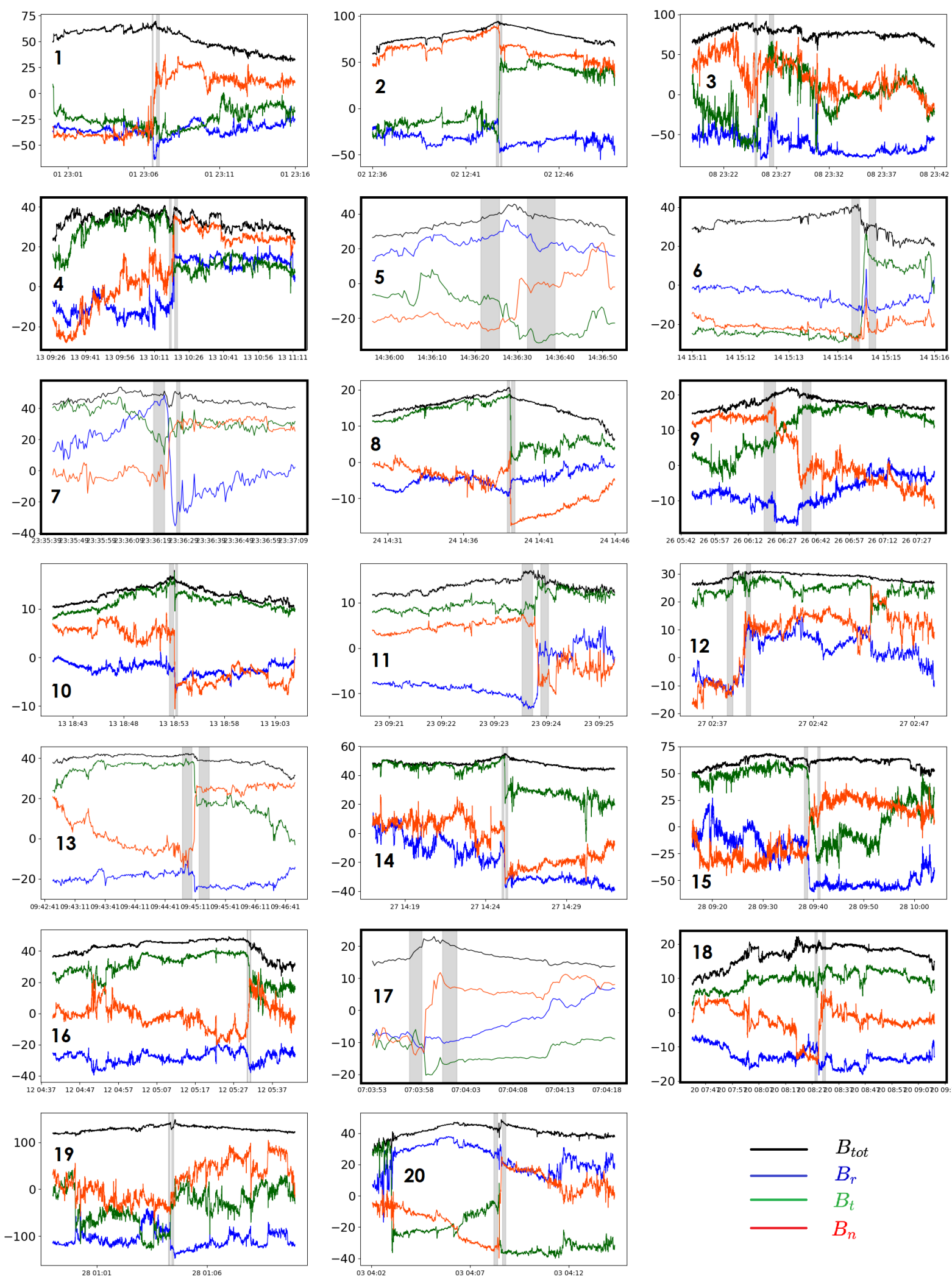

Fig. 5. MICCS magnetic field profile, numbered as in Table 3, with the magnetic field amplitude in black, radial in blue, tangential in green, and normal in red. The shaded areas indicate the intervals used to determine the central current sheet orientation.

median method is biased when PSP is located in the HCS or in a magnetic hole, so we investigated these particular time intervals visually to detect possible MICCS. In particular, the second event analyzed in detail in Sect. 4 was found this way. We also acknowledge that the running median method of detection tends to miss really small events in the solar wind. This does not preclude their existence as indeed some were detected in the HCS crossing of encounter 1 with a duration below $5 \mathrm{~min}$, as observed in the list provided in Table 3. On the other hand, events lasting longer than $6 \mathrm{~h}$ were not detected, but such cases typically would 

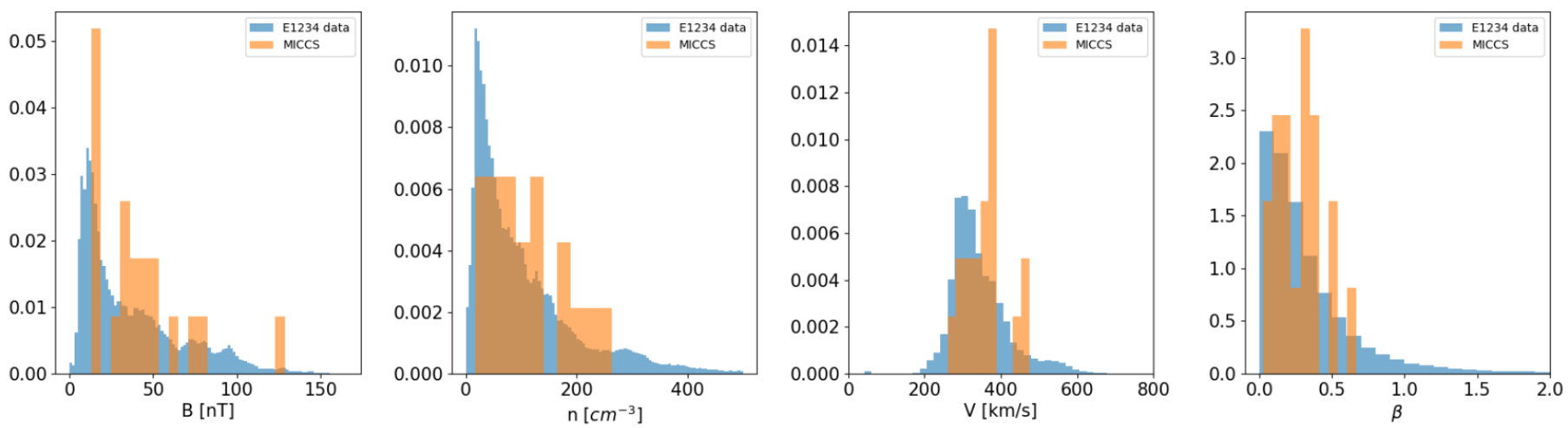

Fig. 6. Density distributions of MICCS properties in the solar wind. Data from the whole period of observation (E1, E2, E3, E4) are displayed in blue and the distribution corresponding to the MICCS observation is in orange.

Table 3. General characteristics of MICCS events.

\begin{tabular}{|c|c|c|c|c|c|c|c|c|c|c|c|}
\hline \# & Enc & Start time & End time & Duration (min) & $\Delta B(\%)$ & Context & $\mathrm{R}\left(R_{\mathrm{S}}\right)$ & $\mathrm{B}(n T)$ & $n\left(\mathrm{~cm}^{-3}\right)$ & $V\left(\mathrm{~km} \mathrm{~s}^{-1}\right)$ & $\beta$ \\
\hline 1 & 1 & 01/11/2018 23:00 & 01/11/2018 23:16 & 16 & 63 & Solar wind & 46.91 & 52 & 263 & 329 & 0.50 \\
\hline 2 & 1 & 02/11/2018 12:36 & 02/11/2018 12:50 & 14 & 45 & Solar wind & 44.48 & 79 & 186 & 321 & 0.11 \\
\hline 3 & 1 & 08/11/2018 23:19 & 08/11/2018 23:42 & 23 & 37 & Solar wind & 41.42 & 76 & 183 & 438 & 0.30 \\
\hline 4 & 1 & 13/11/2018 09:27 & 13/11/2018 11:12 & 105 & 71 & nearby HCS & 61.67 & 33 & 222 & 353 & 0.28 \\
\hline 5 & 1 & 14/11/2018 14:35 & 14/11/2018 14:36 & 1 & 67 & nearby HCS & 67.36 & 35 & 124 & 375 & 0.67 \\
\hline 6 & 1 & 14/11/2018 15:11 & 14/11/2018 15:16 & 5 & 57 & nearby HCS & 67.49 & 31 & 99 & 380 & 0.37 \\
\hline 7 & 1 & 14/11/2018 23:35 & 14/11/2018 23:37 & 1 & 31 & nearby HCS & 69.17 & 45 & 73 & 475 & 0.37 \\
\hline 8 & 1 & $24 / 11 / 2018$ 14:30 & 24/11/2018 14:46 & 16 & 111 & Solar wind & 110.28 & 15 & 17 & 464 & 0.15 \\
\hline 9 & 1 & 26/11/2018 05:47 & 26/11/2018 07:35 & 108 & $40 \%$ & nearby HCS & 116.34 & 18 & 18 & 379 & 0.02 \\
\hline 10 & 2 & $13 / 03 / 2019$ 18:41 & $13 / 03 / 2019$ 19:05 & 24 & $70 \%$ & Solar wind & 123.31 & 12 & 33 & 288 & 0.33 \\
\hline 11 & 2 & 23/03/2019 09:20 & 23/03/2019 09:25 & 5 & $42 \%$ & Solar wind & 86.28 & 14 & 45 & 306 & 0.51 \\
\hline 12 & 2 & 27/03/2019 02:36 & 27/03/2019 02:48 & 12 & $35 \%$ & Solar wind & 69.13 & 29 & 60 & 388 & 0.20 \\
\hline 13 & 3 & 24/08/2019 09:42 & 24/08/2019 09:46 & 4 & $30 \%$ & Solar wind & 66.75 & 39 & 87 & 353 & 0.21 \\
\hline 14 & 3 & $27 / 08 / 2019$ 14:17 & 27/08/2019 14:32 & 15 & $30 \%$ & Solar wind & 51.32 & 48 & 116 & 353 & 0.20 \\
\hline 15 & 3 & 28/08/2019 09:16 & 28/08/2019 10:04 & 48 & $52 \%$ & Solar wind & 47.62 & 61 & 85 & 388 & 0.13 \\
\hline 16 & 3 & 12/09/2019 04:40 & 12/09/2019 05:43 & 63 & 74 & Solar wind & $\ldots$ & 42 & $\ldots$ & $\ldots$ & $\ldots$ \\
\hline 17 & 4 & 19/01/2020 07:03 & 19/01/2020 07:04 & 0,4 & 64 & nearby HCS & 76.82 & 17 & 44 & 345 & 0.23 \\
\hline 18 & 4 & 20/01/2020 07:42 & 20/01/2020 09:13 & 91 & 82 & nearby HCS & 71.42 & 17 & 91 & 260 & 0.31 \\
\hline 19 & 4 & $28 / 01 / 202000: 59$ & 28/01/2020 01:10 & 11 & 30 & Solar wind & 30.28 & 129 & 139 & 374 & 0.05 \\
\hline 20 & 4 & 03/02/2020 04:02 & 03/02/2020 04:14 & 12 & 36 & Solar wind & 47.69 & 41 & 213 & 301 & 0.37 \\
\hline
\end{tabular}

more likely correspond to large-scale structures, such as CMEs or CIRs.

Overall, 20 structures correspond to the criteria (including the ones detailed in Sects. 3 and 4), their general properties are listed in Table 3, and their magnetic profile is shown in Fig. 5. Among them, 13 were detected in the regular slow solar wind and 7 during or near HCS crossings (in bold in Table 3 and with a bold frame in Fig. 5). Table 4 displays the result of some quantitative analysis we performed on the central current sheet and the surrounding switchbacks.

\subsection{General properties}

The MICCS events reported in this paper last between $26 \mathrm{~s}$ and $111 \mathrm{~min}$. For the whole set of events, the mean duration is $29 \mathrm{~min}$, while the median duration is $15 \mathrm{~min}$, and most events last between $5 \mathrm{~min}$ (first quartile) and $42 \mathrm{~min}$ (third quartile). It is interesting to note that all MICCS that were detected nearby HCS crossings are at the tails of the duration distribution, with extreme values over $91 \mathrm{~min}$ or below $5 \mathrm{~min}$. On the other hand, the other MICCS have more homogeneous durations mainly between 12 and $24 \mathrm{~min}$ (mean $20 \mathrm{~min}$, median $15 \mathrm{~min}$ ). As noted in Sect. 5.1, it is possible that some events below $5 \mathrm{~min}$ were missed by our automatic detection method in the solar wind. The relative increase in the magnetic field amplitude goes from $30 \%$ at minimum (by selection) to $111 \%$ at maximum, with a mean increase of $53 \%$. This does not significantly differ, regardless of the context (regular slow solar wind or HCS).

In Fig. 6 we display the density distribution of $B(6 a), n(6 \mathrm{~b})$, $V(6 \mathrm{c})$, and $\beta$ (6d) for MICCS (averaged values). For comparison, the distribution of the whole period of observation where we searched for MICCS structures is displayed in blue, and the MICCS distribution is over plotted in orange. The values are also available in Table 3 for each event. Overall, the MICCS have standard parameters for the solar wind, which follows the distribution for the complete period of observation. Their magnetic field strength goes from 12 to $129 \mathrm{nT}$ and their density from 17 to $222 \mathrm{~cm}^{-3}$. They are mainly detected in the slow solar wind with speeds ranging from 260 to $475 \mathrm{~km} \mathrm{~s}^{-1}$. Their $\beta$ parameter goes from 0.05 to 0.67 .

Figure 7 displays the locations of the observed events for encounters 1 to 4 . The represented frame is the heliocentric 


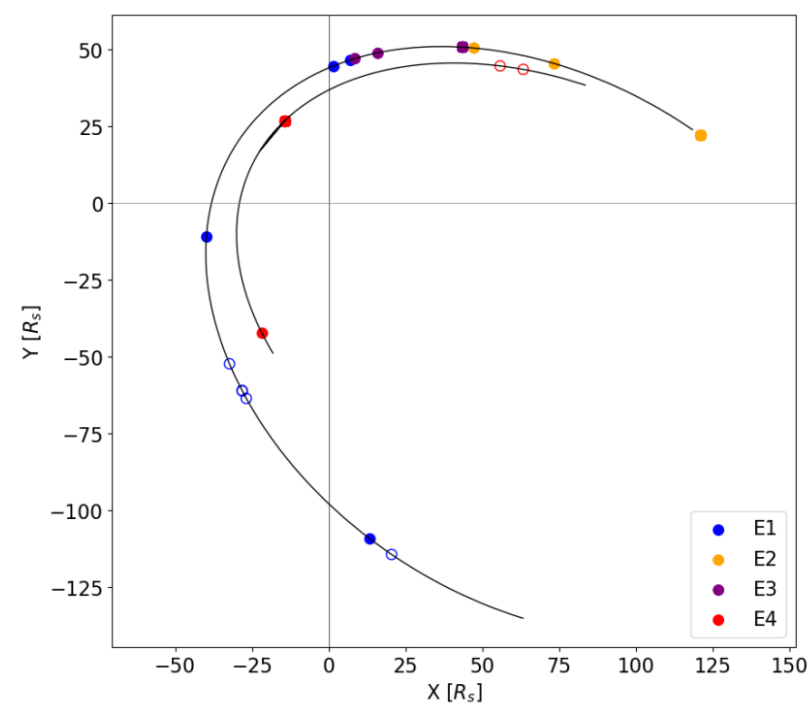

Fig. 7. Detected MICCS positions represented in the heliocentric inertial system for encounters 1 to 4 . Full dots represent MICCS that were detected in the regular slow solar wind, while empty dots are MICCS that were detected near an HCS crossing

inertial system with the XY plane being the ecliptic. The first three PSP orbits follow the same trajectory, while the fourth approaches closer to the Sun. Hollow circles mark the locations of MICCS detected near HCS crossings, which were only observed in encounters 1 and 4. Overall the MICCS are quite evenly distributed along the orbit of PSP and they do not display obvious spatial clustering.

\subsection{Central current sheets orientation}

To determine the main orientation of the events' current sheet, we used the hybrid minimum variance analysis described in Sect. 3.2 and ensured that the direction of maximum variation was reliably determined by checking the ratio of maximum to intermediate eigenvalue. For all current sheets, the ratio ranges from 3.5 to 88.6 , with only one value below 5 . The mean ratio is 30 and the median ratio is 23 . The boundary magnetic fields used in the method were averaged on each side of the current sheets on time spans indicated as gray shaded areas in Fig. 5. The results are displayed in Fig. 8 and show that the central current sheets of MICCS display a preferential orientation.

The top panels represent the $\boldsymbol{l}$ vector (in blue) of the current sheets projected into the RT (Fig. 8a), RN (Fig. 8b), and TN (Fig. 8c) plane. Each projected $\boldsymbol{l}$ vector is plotted as an arrow, with the bold black outer circle representing a norm of 1 . In other words, if an arrow reaches the unit circle in the RT plane, then it has no component in the $\mathrm{N}$ direction. On the contrary, if the norm of the projected arrow is significantly smaller than unity, then its component in the out-of-plane direction is non-negligible. On top of the arrows, a polar bar chart is displayed, counting the number of arrows lying in each direction of the considered plane, with a binning of 22.5 degrees. We distinguish between events observed in the regular slow solar wind (dark blue) from events near HCS crossings (light blue). The bottom panel shows the $\boldsymbol{n}$ vectors in the same manner, with regular slow solar wind events in dark red and those near HCS crossings in magenta.

What stands out in these distributions, and what is also visible when carefully studying Fig. 5, is that the direction of $l$ corresponding to the maximum variance is mainly located within the TN plane of the RTN frame. Figures $8 a$ and $b$, we can see that even if some vectors have significant $\mathrm{R}$ components, none of them are mainly oriented along $\mathrm{R}$, that is to say with a norm close to unity along the $\mathrm{R}$ axis except for one HCS event that has an important $\mathrm{R}$ component (event 7 in Table 3). This preferential orientation is also visible in the polar bar plot, where we can see that most of the $\boldsymbol{l}$ vectors are observed within \pm 45 degrees of the $\mathrm{T}$ axis (12 out of 20) when projected in the RT plane; and this is even higher for the $\mathrm{N}$ axis (16 out of 20) when projected in the $\mathrm{RN}$ plane. This is also confirmed when we look at the projections of $\boldsymbol{l}$ in the TN plane (Fig. 8c) where almost all vectors have a norm close to unity. The bottom panels displays the $\boldsymbol{n}$ vectors, and they are mostly oriented along R. In the RT plane (Fig. 8d), 13 events are within \pm 45 degrees of the $\mathrm{R}$ axis while this is the case for 15 events in the RN plane (Fig. 8e). In the TN plane (Fig. 8f), we can see no preferential orientation, but we notice that almost all vectors have a norm smaller than unity and, as such, present an important $\mathrm{R}$ component. This tendency for the current sheets to be oriented in a particular direction (i.e., with a normal preferred in the $\mathrm{R}$ direction and a maximum variance in the TN plane) does not change when considering only regular slow solar wind events (in dark blue in Figs. 8a-c and dark red in Figs. 8d-f).

\subsection{Reconnecting current sheets}

To determine if a current sheet was reconnecting, we searched for a reconnection jet in the SPC moments coincidental with the magnetic field rotation. We also checked the consistency with variations in the magnetic field magnitude, ion density, plasma $\beta$, and electron pitch angles. It is unfortunate that for the majority of the studied events, data other than the magnetic field were not sufficiently resolved due to either a duration of the event that was too short or a location that was too far out from perihelion. To make the best of the situation, we checked if the observed current sheets were bifurcated, that is, if the magnetic field presented a step in its rotation. This information is a hint as to the magnetic reconnection, as it is a necessary but not sufficient condition (e.g., Phan et al. 2020). Results are presented in Col. 4 (jet) and 5 (bifurcated CS) of Table 4.

Among the 20 current sheets studied, we identified 5 that were associated with a reconnection jet plainly visible in the ion velocity, including the cases described in Sects. 3 and 4. For two events, the diagnosis is less clear and we have marked them as probable jets. For three of them, no jet was visible and the structure was alfvénic throughout. The fact that five out of ten resolved events present a clear reconnection jet hints that MICCS might be a preferential location for magnetic reconnection. Indeed, reconnecting current sheets are not common in PSP measurements, as noted by Phan et al. (2020), because most of the current sheets are located at the boundary of switchbacks. Switchback boundaries are alfvénic structures that propagate in the same direction, contrary to reconnection exhausts, which are bound by two rotational-type discontinuities propagating in opposite directions away from the X-line. In addition, at least four of the remaining unresolved current sheets are clearly bifurcated. They thus potentially reconnect, although we cannot be sure as alfvénic structures can also have bifurcated current sheets, as shown by Phan et al. (2020).

\subsection{Pitch angle distribution}

We investigated the PAD of supra-thermal electrons throughout the structures, as they are good tracers of the large-scale magnetic topology. We visually determined if the PAD showed sig- 

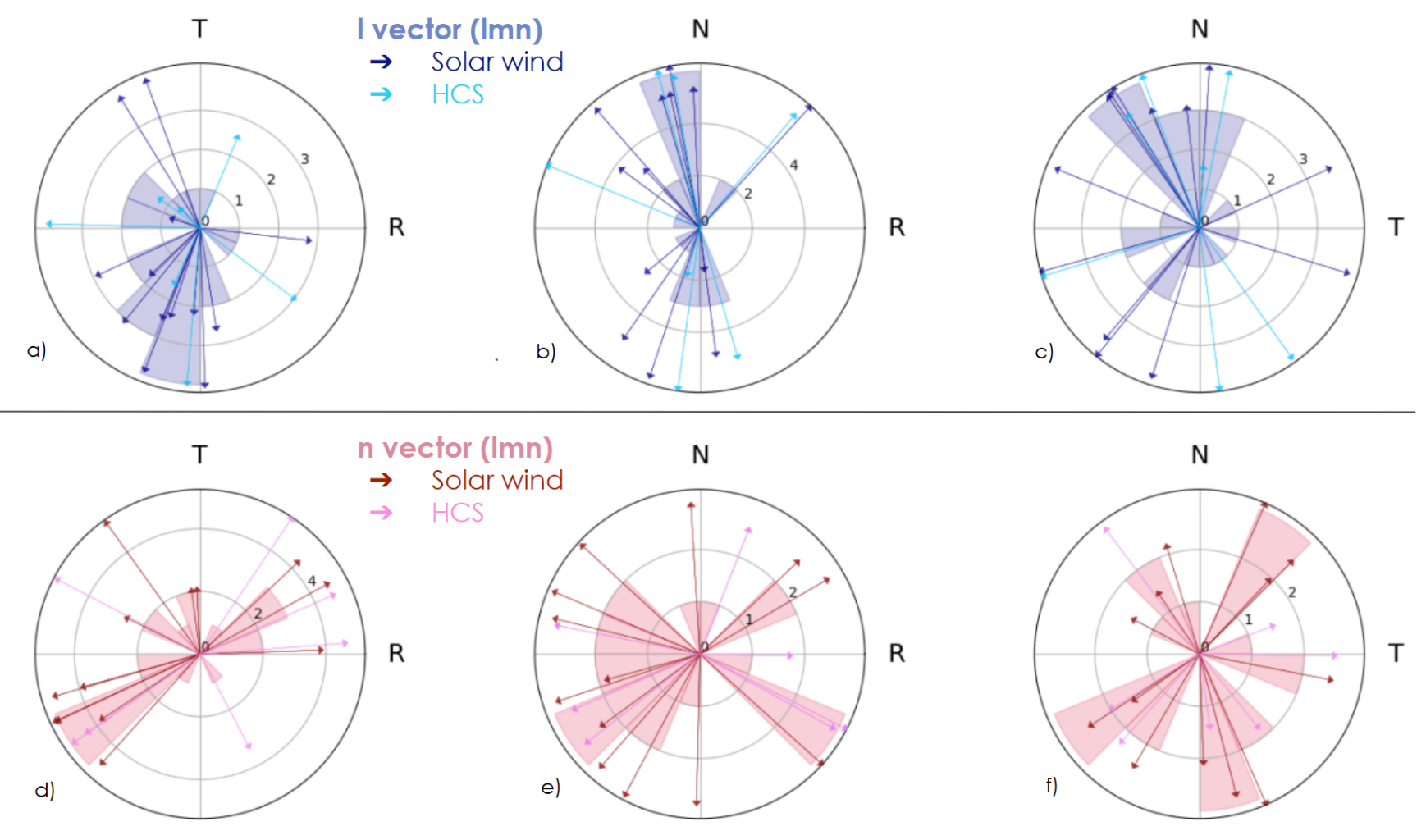

Fig. 8. MICCS central current sheet lmn orientation. Top panels: $\boldsymbol{l}$ vectors projected in the RT (left), RN (center), and TN (right) plane, with a solar wind event in dark blue and HCS events in light blue. A polar bar chart is over plotted counting the number of arrows in a $22.5^{\circ}$ bin. Bottom panels: $\boldsymbol{n}$ vectors in a similar manner, with a solar wind event in dark red and HCS events in magenta. See text for more details.

Table 4. Quantitative analysis on MICCS events.

\begin{tabular}{|c|c|c|c|c|c|c|}
\hline$\#$ & Start time & Duration (min) & Jet & Bifurcated CS & PAD variation & Switchbacks \\
\hline 1 & 01/11/2018 23:00 & 16 & No & No & Yes & Yes \\
\hline 2 & 02/11/2018 12:36 & 14 & Yes & Yes & No & Yes \\
\hline 3 & 08/11/2018 23:19 & 23 & No & No & Yes & Yes \\
\hline 4 & 13/11/2018 09:27 & 105 & Yes & Yes & Yes & $\ldots$ \\
\hline 5 & $14 / 11 / 201814: 35$ & 1 & Not resolved & Maybe & Not resolved & $\ldots$ \\
\hline 6 & 14/11/2018 15:11 & 5 & Not resolved & No & Not resolved & ... \\
\hline 7 & $14 / 11 / 2018$ 23:35 & 1 & Not resolved & Maybe & Not resolved & $\ldots$ \\
\hline 8 & 24/11/2018 14:30 & 16 & Not resolved & Yes & Not resolved & Yes \\
\hline 9 & 26/11/2018 05:47 & 108 & Not resolved & Yes & Unclear & $\ldots$ \\
\hline 10 & 13/03/2019 18:41 & 24 & Not resolved & Yes & Not resolved & Yes \\
\hline 11 & 23/03/2019 09:20 & 5 & Not resolved & Yes & Not resolved & Yes \\
\hline 12 & $27 / 03 / 201902: 36$ & 12 & Not resolved & Maybe & Not resolved & Yes \\
\hline 13 & 24/08/2019 09:42 & 4 & Yes & Yes & No & Yes \\
\hline 14 & $27 / 08 / 201914: 17$ & 15 & Probable jet & Yes & Yes & Yes \\
\hline 15 & 28/08/2019 09:16 & 48 & Yes & Yes & Yes & Yes \\
\hline 16 & $12 / 09 / 201904: 40$ & 63 & No data & Maybe & Not resolved & Yes \\
\hline 17 & 19/01/2020 07:03 & 0.4 & Not resolved & Maybe & Not resolved & $\ldots$ \\
\hline 18 & $20 / 01 / 202007: 42$ & 91 & No & No & Unclear & $\ldots$ \\
\hline 19 & 28/01/2020 00:59 & 11 & Probable jet & Maybe & No & No \\
\hline 20 & 03/02/2020 04:02 & 12 & Yes & Yes & No & Yes \\
\hline
\end{tabular}

nificant variations, upstream and downstream of the current sheet (and within the structure's boundaries), focusing on changes in the maximum flux location, a broadening of the PAD distribution, and the presence of counter-streaming electrons. The results are presented in the PAD variation column of Table 4. Five events present clear variations in their strahl properties across the current sheet (indicated by a yes in Table 4), while others are either unresolved or unclear, and four of them rather clearly show no variation (indicated by a no). Of the four events with a PAD variation, only the one described in Sect. 4 (\#4) is located near the HCS, while the four other events $(\# 1,3,14,16)$ are located in the regular slow solar wind. The four events with no variation of PAD $(\# 2,15,19,20)$ are also located in the regular slow solar wind and present no particular feature in strahl properties. 
In the four events with a PAD variation, a counter-streaming electron population is present in only one part of the structure and vanishes across the current sheet, as in Fig. 3g. Given their often much lower fluxes, it is not clear whether these counterstreaming electrons are indicative of closed field lines, or if they may come from reflections farther down the field lines by some distant CIR or shock (Steinberg et al. 2005, Skoug et al. 2006, Lavraud et al. 2010). Yet close to the HCS, closed field lines may form more easily, as flux ropes of various sizes with clearly closed field lines have been observed frequently in that region up to $1 \mathrm{AU}$ (e.g., Kilpua et al. 2009, Rouillard et al. 2011, SanchezDiaz et al. 2019). As such, closed field lines during the first part of event \#4 (Sect. 4) remain a plausible configuration. But in either case, it is clear that for these five events (\#1,3,4,14,16), the large-scale connectivity of the field lines before and after the current sheet is drastically different.

\subsection{Relation to switchbacks}

As the first event analyzed in Sect. 3 was observed near switchbacks, we checked whether this connection was true for all MICCS. In Col. 7 of Table 4, we indicated if the regular slow solar wind events were located in the vicinity of switchbacks (abbreviated SB) or rather with no switchback around. Events located near HCS events were not considered here. Most of the regular slow solar wind events were observed in close relation to a switchback except for one (\#19). But we can see no clear tendency for them to be before or after, or a direct part of the switchbacks. As switchbacks are omnipresent in PSP data, the proximity of the MICCS structure and switchbacks is as likely to be random as it is to be significant. Additional work is required to conclude on the possible link between these two types of structures.

\subsection{PSP dust measurements}

One model that has been proposed to explain the formation of similar magnetic peaks in the solar wind is the dust-plasma interaction process studied with regards to IFE formation (Russell 1990, Jones et al. 2003a,b). Here, we analyze PSP dust measurements made by the FIELDS instrument at the time of observation of the MICCS structures. Dust, when impacting the spacecraft, creates voltage spikes on the electric antenna with amplitudes well above the instrument noise level (Szalay et al. 2020, Page et al. 2020). We looked at the voltage provided by the second antenna of PSP (V2) and analyzed the number of spikes superior to $50 \mathrm{mV}$ (as done in Szalay et al. 2020) with a binning of $5 \mathrm{~min}$, considering this quantity to be a good approximation for the number of dust impacts on the spacecraft. As an illustration, this number of spikes is plotted on the top panel of Fig. 9 for the event described in Sect. 4. We computed the average and standard deviation of the number of spikes on a $\pm 3 \mathrm{~h}$ interval outside of the event (red curve and shaded area in Fig. 9a) and compared it to the average inside the event (blue curve in Fig. 9a). The latter was computed with a \pm 5 min interval around the MICCS to be sure that we obtained at least two points for the shorter events.

We then computed these quantities for all the MICCS under study; the results for all events are displayed in the bottom panel of Fig. 9. We find that for all events, except for one (\#6), the average number of spikes inside the MICCS falls within $1 \sigma$ of the outside average. We conclude that there is no significant change in the dust impact rate (through the number of spikes) in the vicinity of the MICCS events studied here.

\section{Formation models}

In the Introduction, we have presented two models that may explain the formation of these magnetic peaks with central current sheet in the solar wind. Regarding the dust-plasma interaction process studied with regards to IFE formation (Russell 1990, Jones et al. 2003a,b), we investigated PSP dust measurements in Sect. 5.7 and found no significant change in the dust impact rate measured by PSP at the time of observation of the MICCS. The most probable conclusion is that dust plays no role in the formation and evolution of the MICCS structures. However, it is possible that dust plays a role in their formation while its signature has faded over time, or that the population of dust grains responsible for the MICCS formation has sizes and velocities that are not detected by PSP. Measurements by Solar Orbiter will prove interesting to further confirm or contradict our result.

On the other hand, at the Earth's magnetopause, FTE-like structures with a magnetic reconnection in their core were identified as interlinked flux tubes. Following this view, the MICCS structures may also be interlaced flux tubes embedded in and advected by the solar wind. The fact that PADs vary across the current sheets for several events is consistent with this view of two flux tubes connected to different strahl source regions, as discussed in Sect. 5.5.

At the Earth's magnetopause, it was argued that an IMF strongly tilted duskward or dawnward would create complex configurations at the subsolar point with several X-lines, and thus facilitate the creation of interlaced flux tubes (Fargette et al. 2020). In the solar wind, the flux tubes on each side of the current sheet may well originate from very distinct sources on the photosphere (explaining the often very different properties of the flux tubes) and be transported in the heliosphere where the 3D expansion of the solar wind may allow for their interaction. This would be consistent with the tendency for the current sheets to have a normal - that is a minimum variation direction - along R. Indeed, in the flux tube texture view of Borovsky (2008), the flux tubes that constitute the solar wind remain rooted to the photosphere despite often forming strong current sheets at their boundaries and potentially entangling themselves significantly. A strong interaction between two flux tubes may lead to an increased magnetic field and interlacing such that the plane of interaction may preferentially be perpendicular to the main direction of the flow, thus explaining the preferred orientation of the current sheet normal vectors with the radial direction.

The MICCS observed near the HCS easily fit in this scenario. They may even be more frequent since the HCS is a location where it is even easier to form adjacent flux tubes with drastically different magnetic connectivity.

Regarding the relation to switchbacks, one may easily imagine that SBs can entangle with other SBs or with the background solar wind to form complex structures such as MICCS. Yet at this point, the apparent frequent vicinity of SBs appears marginal given the large recurrence of SBs in PSP data overall.

\section{Conclusion}

In this work, we have performed a systematic study of structures made of a magnetic field increase with a clear central current sheet, resembling solar wind interplanetary field enhancements (IFEs) or magnetospheric interlinked flux tubes (IFTs), observed by the Parker Solar Probe mission and below 0.3 AU. We first reported on two of these events in detail. One occurred in the 

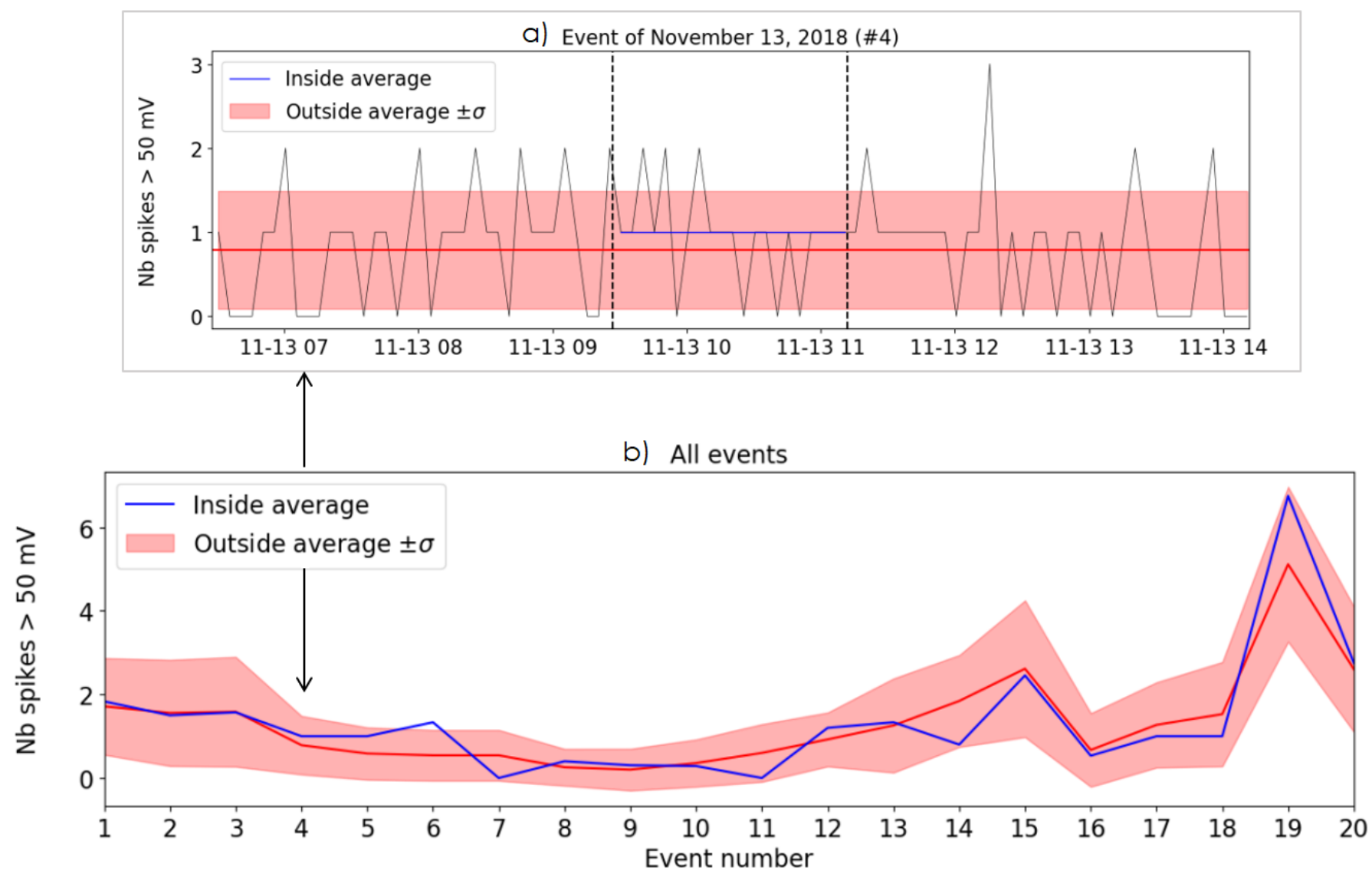

Fig. 9. Analysis of PSP dust measurements in the vicinity of MICCS. (a) Number of voltage spikes superior to $50 \mathrm{mV}$ from the V2 antenna as a function of time, around the event described in Sect. 4 (delimited with dashed lines). The red line is the average of the number of spikes on a $\pm 3 \mathrm{~h}$ interval around the MICCS, and the red shaded area delimits a $1 \sigma$ standard deviation. The blue line is the average inside the structure. $(b)$ Same statistical quantities for all events. The red curve is the averaged number of spikes for each event surrounded by the $\pm 1 \sigma$ shaded area, and the blue curve is the average number of spikes inside each MICCS.

regular slow solar wind and the other was near an HCS crossing, and both displayed reconnection signatures at their central current sheet.

We then identified 20 MICCS detected by PSP throughout its first four encounters with the Sun. They were selected based on an increase in the magnetic field strength over $30 \%$ and the presence of a central current sheet. The following points summarize our results.

- We find that these structures can be detected in the regular slow solar wind (13 events) as well as during HCS crossings (7 events) at all longitudes and distances from the Sun.

- Their general characteristics are standard, with $B, n, V$, and $\beta$ values consistent with the slow solar wind, and with a maximum speed of $475 \mathrm{~km} \mathrm{~s}^{-1}$. The PSP orbit, potential bias in our selection method, and the current low statistics do not allow us to be conclusive yet, but as of now these structures have not been observed in the fast solar wind by PSP.

- They last on average $29 \mathrm{~min}$, with extreme durations from less than a minute to close to $2 \mathrm{~h}$. The extreme durations are often associated with HCS events. MICCS in the regular slow solar wind are more likely to last between 12 and 24 min.

- Several of the central current sheets showed reconnection jets (five identified, two probable) and/or bifurcated magnetic field signatures (ten identified) in their core. But many cases had insufficient data resolution to determine if reconnection was in fact occurring.
- For five events, the supra-thermal electron PAD varies across the central current sheet, hinting that there is a significant change in connectivity between the first and second part of the events, as delimited by the central current sheet. For four events, the PAD shows no variation suggesting no significant change in connectivity to the Sun.

- The central current sheets display a preferential orientation, with a maximum variance direction in the TN plane of the RTN frame and a normal oriented in the R direction.

- When detected in the regular slow solar wind, the structures are often close to switchbacks. However, considering the omnipresence of switchbacks in PSP data, it is difficult to draw a significant conclusion as to a possible relation.

- We find no significant change in the dust impact rate in the vicinity of the MICCS under study, leading us to conclude that dust probably plays no role in the MICCS formation and evolution.

Overall our observations are consistent with MICCS being constituted of two entangled flux tubes embedded in the solar wind plasma that interact at their current sheet boundary. The flux tubes would remain rooted at the Sun as indicated by the preferential orientation of their current sheet in the TN plane and PAD properties within the structure. Some show significant strahl variation across their current sheet and we conclude that they are either connected to different sources back at the Sun on one end, or that they extend to different regions in the heliosphere on the other end (CIRs, shocks). Magnetic reconnection is frequently 
observed at their current sheet, which makes them a favored place in which to search and study this process as we approach closer to the Sun.

Future observations by PSP of such structures with a higher time resolution and those that are lower in the corona will unveil more about their properties and formation process. A combined observation with the Solar Orbiter mission (Müller et al. 2020) will also enable detailed studies regarding their early evolution.

Acknowledgements. We acknowledge the NASA Parker Solar Probe Mission and particularly the FIELDS team led by S. D. Bale and the SWEAP team led by

J. Kasper for use of data. Parker Solar Probe was designed, built, and is now operated by the Johns Hopkins Applied Physics Laboratory as part of NASA's Living with a Star (LWS) program (contract NNN06AA01C). Support from the LWS management and technical team has played a critical role in the success of the Parker Solar Probe mission. The data used in this study are available at the NASA Space Physics Data Facility (SPDF): https://spdf.gsfc.nasa.gov, and at the PSP science gateway https://sppgway.jhuapl.edu/. We visualize data using the CLWeb software available at http: //clweb. irap. omp. eu/, developed by E. Penou. Work at IRAP was supported by CNRS, CNES and UPS. J.P.E. is supported by UKRI/STFC grant ST/S000364/1. The author N.F.A. acknowledges the support of the ISSI team, working in unraveling solar wind microphysics in the inner heliosphere.

\section{References}

Antiochos, S. K., Mikić, Z., Titov, V. S., Lionello, R., \& Linker, J. A. 2011, ApJ, 731,112

Badman, S. T., Bale, S. D., Martínez Oliveros, J. C., et al. 2020, ApJS, 246, 23

Bale, S. D., Goetz, K., Harvey, P. R., et al. 2016, Space Sci. Rev., 204, 49

Bale, S. D., Badman, S. T., Bonnell, J. W., et al. 2019, Nature, 576, 237

Borovsky, J. E. 2008, J. Geophys. Res. Space Phys., 113, A08110

Bruno, R., \& Carbone, V. 2013, Liv. Rev. Sol. Phys., 10, 2

Cardoso, F. R., Gonzalez, W. D., Sibeck, D. G., Kuznetsova, M., \& Koga, D. 2013, Ann. Geophys., 31, 1853

Case, A. W., Kasper, J. C., Stevens, M. L., et al. 2020, ApJS, 246, 43

Cranmer, S. R. 2009, Liv. Rev. Sol. Phys., 6, 3

Dudok de Wit, T., Krasnoselskikh, V. V., Bale, S. D., et al. 2020, ApJS, 246, 39

Fargette, N., Lavraud, B., Øieroset, M., et al. 2020, Geophys. Res. Lett., 47, e86726

Farinas Perez, G., Cardoso, F. R., Sibeck, D., et al. 2018, J. Geophys. Res. Space Phys., 123, 1337

Gosling, J. T., \& Phan, T. D. 2013, ApJ, 763, L39

Gosling, J. T., Borrini, G., Asbridge, J. R., et al. 1981, J. Geophys. Res., 86, 5438

Haggerty, C. C., Shay, M. A., Chasapis, A., et al. 2018, Phys. Plasmas, 25, 102120

Hesse, M., Birn, J., \& Schindler, K. 1990, J. Geophys. Res., 95, 6549

Horbury, T. S., Woolley, T., Laker, R., et al. 2020, ApJS, 246, 45
Hudson, P. D. 1970, Planet. Space Sci., 18, 1611

Jones, G. H., Balogh, A., McComas, D. J., \& MacDowall, R. J. 2003a, Icarus, 166,297

Jones, G. H., Balogh, A., Russell, C. T., \& Dougherty, M. K. 2003b, ApJ, 597, L61

Kacem, I., Jacquey, C., Génot, V., et al. 2018, J. Geophys. Res. Space Phys., 123, 1779

Kasper, J. C., Abiad, R., Austin, G., et al. 2016, Space Sci. Rev., 204, 131

Kasper, J. C., Bale, S. D., Belcher, J. W., et al. 2019, Nature, 576, 228

Kilpua, E. K. J., Luhmann, J. G., Gosling, J., et al. 2009, Sol. Phys., 256, 327

Lai, H. R., Russell, C. T., Jia, Y. D., Wei, H. Y., \& Angelopoulos, V. 2015, Geophys. Res. Lett., 42, 1640

Lapenta, G., \& Knoll, D. A. 2005, ApJ, 624, 1049

Lavraud, B., Opitz, A., Gosling, J. T., et al. 2010, Ann. Geophys., 28, 233

Lavraud, B., Fargette, N., Réville, V., et al. 2020, ApJ, 894, L19

Lee, L. C., \& Fu, Z. F. 1985, Geophys. Res. Lett., 12, 105

Liu, Y.-H., Drake, J. F., \& Swisdak, M. 2011, Phys. Plasmas, 18, 092102

Louarn, P., Fedorov, A., Budnik, E., et al. 2004, Geophys. Res. Lett., 31, L19805

Mann, I., Czechowski, A., \& Meyer-Vernet, N. 2010, AIP Conf. Ser., 1216, 491

Müller, D., St. Cyr, O. C., Zouganelis, I., et al. 2020, A\&A, 642, A1

Nishida, A. 1989, Geophys. Res. Lett., 16, 227

Øieroset, M., Phan, T. D., Haggerty, C., et al. 2016, Geophys. Res. Lett., 43, 5536

Øieroset, M., Phan, T. D., Drake, J. F., et al. 2019, Geophys. Res. Lett., 46, 1937

Otto, A. 1995, J. Geophys. Res., 100, 11863

Page, B., Bale, S. D., Bonnell, J. W., et al. 2020, ApJS, 246, 51

Phan, T. D., Bale, S. D., Eastwood, J. P., et al. 2020, ApJS, 246, 34

Réville, V., Velli, M., Panasenco, O., et al. 2020, ApJS, 246, 24

Rouillard, A. P., Sheeley, N. R. J., Cooper, T. J., et al. 2011, ApJ, 734, 7

Russell, C. T. 1990, Adv. Space Res., 10, 159

Russell, C. T., \& Elphic, R. C. 1979, Geophys. Res. Lett., 6, 33

Russell, C. T., \& Qi, Y. 2020, Geophys. Res. Lett., 47, e87620

Russell, C. T., Arghavani, M. R., \& Luhmann, J. G. 1984, Icarus, 60, 332

Russell, C. T., Weimer, D. R., Omidi, N., et al. 2010a, Geophys. Res. Lett., 37, L07204

Russell, C. T., Jian, L. K., Lai, H. R., et al. 2010b, AIP Conf. Ser., 1216,522

Sanchez-Diaz, E., Rouillard, A. P., Davies, J. A., et al. 2017, ApJ, 851, 32

Sanchez-Diaz, E., Rouillard, A. P., Lavraud, B., Kilpua, E., \& Davies, J. A. 2019, ApJ, 882, 51

Skoug, R. M., Gosling, J. T., McComas, D. J., Smith, C. W., \& Hu, Q. 2006, J. Geophys. Res. Space Phys., 111, A01101

Sonnerup, B. U. O., \& Cahill, L. J. J. 1967, J. Geophys. Res., 72, 171

Sonnerup, B. U. O., Paschmann, G., Papamastorakis, I., et al. 1981, J. Geophys. Res., 86, 10049

Southwood, D. J., Farrugia, C. J., \& Saunders, M. A. 1988, Planet. Space Sci., 36,503

Steinberg, J. T., Gosling, J. T., Skoug, R. M., \& Wiens, R. C. 2005, J. Geophys. Res. Space Phys., 110, A06103

Szabo, A., Larson, D., Whittlesey, P., et al. 2020, ApJS, 246, 47

Szalay, J. R., Pokorný, P., Bale, S. D., et al. 2020, ApJS, 246, 27

Wang, Y. M. 1994, ApJ, 437, L67

Whittlesey, P. L., Larson, D. E., Kasper, J. C., et al. 2020, ApJS, 246, 74 